\title{
Virtual Indicative Broadband over Power Lines Topologies for Respective Subclasses by Adjusting Channel Attenuation Statistical Distribution Parameters of Statistical Hybrid Models (Class Maps) - Part 2: Numerical Results for the Overhead and Underground Medium-Voltage Power Grids
}

\author{
Athanasios G. Lazaropoulos* \\ School of Electrical and Computer Engineering / National Technical University of Athens / \\ 9 Iroon Polytechniou Street / Zografou, GR 15780
}

Received June 21, 2019; Accepted August 12, 2019; Published August 16, 2019

\begin{abstract}
With reference to the initial statistical hybrid model (iSHM) and modified statistical hybrid model (mSHM), the theory of the definition procedure of new virtual indicative distribution BPL topologies by appropriately adjusting the channel attenuation statistical distributions (CASDs) parameters of iSHM and $\mathrm{mSHM}$ has been presented in [1]. In this paper, the results of the definition procedure for the OV and UN MV BPL networks are first presented through the prism of the proposed class maps of iSHM and mSHM.
\end{abstract}

Keywords: Smart Grid; Broadband over Power Lines (BPL) networks; Power Line Communications (PLC); Distribution Power Grids; Capacity; Statistics; Modeling

\section{Introduction}

This set of papers deals with two fervent issues of the broadband over power lines (BPL) statistical channel modelling, say: (i) the underrepresentation of the BPL topology classes during the BPL statistical channel modelling through the insertion of virtual indicative BPL topologies and their respective subclasses; and (ii) the graphical representation of the BPL topology classes and subclasses in terms of their average capacity through the proposed class maps of the first paper [1]. First, as the BPL statistical channel modelling is concerned, two versions of the hybrid statistical model have already been proposed, say the initial statistical hybrid model (iSHM), which has been presented in [2], [3], and the modified statistical hybrid model (mSHM), which has been presented in [4]. The basic component of both hybrid statistical models is the deterministic hybrid model that has exhaustively been validated in a plethora of transmission and distribution BPL network cases [5]-[15]. Apart from the deterministic hybrid model, iSHM applies five well-known channel attenuation statistical distribution (CASDs) of the communications literature, say, Gaussian, Lognormal, Wald, Weibull and Gumbel ones [2], [3], while mSHM applies the Empirical CASD [4] so that BPL topology classes -i.e., rural, suburban, urban and aggravated urban subclass- and 
respective subclasses can be defined. Second, as the underrepresentation of the BPL topology classes is concerned, by appropriately adjusting the CASD parameters of iSHM and mSHM -i.e., CASD maximum likelihood estimators (MLEs) and Empirical cumulative density function (CDF) for iSHM and mSHM, respectively-, new virtual indicative distribution BPL topologies can be proposed that are added to the existing real ones and further define their respective distribution BPL topology subclasses enriched with corresponding statistically equivalent BPL topologies. In [1], the theoretical framework for the definition procedure of the virtual indicative BPL topologies and their respective virtual subclasses, briefly denoted as definition procedure hereafter, has been detailed. Third, as the graphical capacity representation of the real and virtual subclasses is concerned, the main output of the definition procedure of [1] is the class map, which is a 2D contour plot for given power grid type, coupling scheme, injected power spectral density (IPSD) limits and noise levels, that is the graphical synthesis of the CASD parameter and subclass map; say, real and virtual distribution BPL subclasses are graphically categorized into suitable BPL topology class areas in terms of their corresponding average capacities where the axes of the contour plot are proper adjustments of CASD parameters of iSHM and mSHM. The numerical results of the full deployment of class mapping are first presented in this paper for overhead medium voltage (OV MV) and underground medium voltage (UN MV) BPL networks while the BPL statistical channel modelling concept along with the class map is first applied to OV high-voltage (HV) BPL networks in [16].

The rest of this paper is organized as follows: Section II synopsizes the default settings that are required for the fine operation of the deterministic hybrid model, iSHM, $\mathrm{mSHM}$ and the definition procedure. In Section III, the numerical results of the definition procedure of iSHM and mSHM are demonstrated on the basis of the class maps of the OV MV and UN MV BPL networks. Section IV concludes this paper.

\section{The Operation Settings of Class Mapping}

To coexist the deterministic hybrid model, iSHM and mSHM with the definition procedure, a set of operation settings, which have already been reported in [2]-[4], should be assumed. In this Section, a synopsis of these assumptions is given as well as the set of scenarios that is studied in this paper.

\subsection{Operation Settings Concerning the Operation of the Deterministic Hybrid Model}

With reference to Fig. 1 of [1], the five indicative OV MV and UN MV BPL topologies, which are reported in Tables 1 and 2 of [1], respectively, are considered as the representative ones of the respective main OV MV and UN MV BPL topology subclasses. Note that with reference to [5], [8], [10], [17], the indicative OV MV and UN MV BPL topologies concern average long end-to-end connections of $1000 \mathrm{~m}$ and $200 \mathrm{~m}$, respectively.

As the the circuital parameters of the above indicative OV MV and UN MV BPL topologies are concerned for the operation of the deterministic hybrid model, these are detailed in [5], [7]-[15], [18]-[33]. Synoptically, the required assumptions can be 
synopsized as follows: (i) the branching cables are assumed identical to the distribution cables; (ii) the interconnections between the distribution and branch conductors are fully activated; say all the phase and the neutral conductors of the branching cables are connected to the respective ones of the distribution cables; (iii) the transmitting and the receiving ends are assumed matched to the characteristic impedance of the modal channels; and (iv) the branch terminations are assumed open circuit since MV/LV transformers are assumed to be installed.

\section{2 iSHM Operation Settings}

Already been identified in [3], the distribution power grid type, the BPL topology class, CASD, electromagnetic interference (EMI) policy, noise level and applied coupling scheme are factors that should be carefully selected during the operation of the deterministic hybrid model and iSHM. Hence, additional details should be given for iSHM operation settings as reported in this subsection.

First, the BPL operation frequency range and the flat-fading subchannel frequency spacing are assumed to be equal to $3-30 \mathrm{MHz}$ and $0.1 \mathrm{MHz}$ (i.e, $f_{s}=0.1 \mathrm{MHz}$ ), respectively. Hence, there are 270 subchannels in the frequency range of interest (i.e., $Q=270$ ). In accordance with [8], [10], [11], [19], [21], [34], the assumption of the flat-fading subchannels, which is a typical scenario, remains a crucial element towards the capacity computation of the examined distribution BPL topologies, that is anyway the output measure of iSHM.

Second, to compute coupling scheme channel attenuations of the distribution BPL topologies, CS2 module, which has been detailed in [35], [36], is applied as the default coupling scheme system of the deterministic hybrid model. Among the available coupling schemes that are supported by $\mathrm{CS} 2$ module, $\mathrm{WtG}^{1}$ and $\mathrm{StP}^{1}$ coupling schemes are assumed to be the default ones for the assessment of OV MV and UN MV BPL topology subclasses, respectively, so that a direct comparison between the results of this paper and those of [3] and [4], can be achieved.

Third, during the computation of the coupling scheme channel attenuation differences in the Phase B of [2], values that are greater or equal to zero are expected in the vast majority of the cases. However, in the scarce cases of negative coupling scheme channel attenuation differences and in "LOS" cases, the coupling scheme channel attenuation differences are assumed to be equal to an arbitrarily low value, say $1 \times 10^{-11}$. This assumption is made in order to prevent the presence of infinite terms due to the natural logarithms and denominators of Lognormal, Wald and Weibull channel attenuation distributions in iSHM.

Fourth, as the members of each BPL topology subclass are concerned in Phase D, 100 member distribution BPL topologies (i.e., $P=100$ ) are assumed to be added in each BPL topology subclass per CASD through the statistical hybrid model procedure described in [2].

Fifth, during the capacity computations of iSHM, FCC Part 15 is considered as the default IPSD limit proposal concerning EMI policies for BPL systems in this paper. Among the available EMI policies, which are FCC Part 15, German Reg TP NB30 and the BBC / NATO Proposal and their impact on the performance of hybrid statistical models has already been studied in [3], [4], FCC Part 15 is assumed to be the default EMI policy in this paper due to its proneness to the broadband character of BPL networks and 
its high performance results of statistical hybrid models. In the frequency range 3-30 $\mathrm{MHz}$ of this paper, $-60 \mathrm{dBm} / \mathrm{Hz}$ and $-40 \mathrm{dBm} / \mathrm{Hz}$ are the FCC Part 15 IPSD limits $p(\cdot)$ suitable for the operation of OV MV and UN MV BPL topologies, respectively [8], [10], [37].

Sixth, during the capacity computations, uniform additive white Gaussian noise (AWGN) PSD levels are assumed in accordance with the FL noise model of [38], [39]. As it regards the noise properties of OV MV and UN MV BPL networks in the $3-30 \mathrm{MHz}$ frequency range [8], [10], [19], [34], [40], $-105 \mathrm{dBm} / \mathrm{Hz}$ and $-135 \mathrm{dBm} / \mathrm{Hz}$ are the appropriate AWGN PSD limit levels $N(\cdot)$ for OV MV and UN MV BPL networks, respectively.

Seventh, in accordance with [3], [4], the performance of iSHM and the accuracy of its capacity results significantly depend on the selection of the CASD. Based on the findings of Table 3 of [3], it has been demonstrated for the iSHM that Weibull and Wald CASDs perform the best capacity estimations in OV MV and UN MV power grid types, respectively, regardless of the examined BPL topology subclass when the aforementioned operation settings concerning EMI policy, noise level and applied coupling scheme are assumed. These two CASDs are going to be only adopted by the following definition procedure and during the iSHM study in this paper.

\section{3 mSHM Operation Settings}

Similarly to iSHM, the distribution power grid type, the BPL topology class, the reference indicative distribution BPL topology, EMI policy, noise level and applied coupling scheme are factors that are involved during the operation of the deterministic hybrid model and mSHM [4]. In contrast with iSHM operation settings, only one CASD, say, the Empirical CASD, is adopted by mSHM by default and, hence, CASD selection is not considered among the mSHM operation settings.

As the BPL operation frequency range, flat-fading subchannel spacing, coupling scheme system, the member number of each BPL topology subclass, EMI policy and noise level are considered, their properties described in Sec. 2.2 for iSHM are assumed to be the same with the ones of mSHM. For comparison reasons between iSHM and mSHM and in accordance with [2], [3], [4], the infinity prevention assumption remains for the $\mathrm{mSHM}$.

In general, each indicative distribution BPL topology is characterized by a set of parameters regarding either iSHM (i.e., MLEs) or mSHM (i.e., Empirical CDF). As already been mentioned in Sec.2.2 for iSHM, Weibull and Wald CASD MLEs perform the best capacity estimations in OV MV and UN MV power grid types, respectively, and these MLEs are going to be adopted for the rest of this paper. As mSHM CASD parameters are concerned, the Empirical CDF of a reference distribution BPL topology among the indicative distribution BPL topologies of the main subclasses acts as the mSHM CASD parameter that is going to be delivered to the following definition procedure.

\subsection{Operation Settings for the Definition Procedure}

In accordance with [1], the BPMN diagrams of iSHM and mSHM flowchart are presented in Figs. 2(a) and 2(b) of [1], respectively. With reference to the aforementioned figures, the additional BPMN elements (i.e., the virtual topology modules and their 
corresponding outputs), which will allow the application of the definition procedure, are shown in red color. Apart from the BPMN diagrams, the interaction of the virtual topology modules with the remaining iSHM and mSHM steps is depicted through the flowcharts of Figs. 3(a) and 3(b) of [1], respectively. By comparing Figs. 3(a) and 3(b) of [1], several differences concerning these flowcharts can be observed, especially: (i) between steps FL1.04 and FL2.04; and (ii) between steps FL1.05 and FL2.05. Due to the previous differences, the operation settings concerning the definition procedure can be divided into two groups, namely:

1. iSHM definition procedure: With reference to [2], [3], since Weibull CASD performs the best capacity estimations in OV MV BPL networks, $\hat{a}_{\mathrm{MLE}}^{\text {Weibull }}$ and $\hat{\beta}_{\mathrm{MLE}}^{\text {Weibull }}$ are Weibull CASD MLEs that are used in FL1.04 of Fig. 3(a) of [1]. When UN MV BPL topologies are studied, $\hat{\mu}_{\mathrm{MLE}}^{\mathrm{Wald}}$ and $\hat{\lambda}_{\mathrm{MLE}}^{\text {Wald }}$ are the Wald CASD MLEs that are used in FL1.04 of Fig. 3(a) of [1]. In accordance with FL1.04 of Fig. 3(a) of [1], MLEs of the indicative distribution BPL topologies of main subclasses are computed. To compute the MLE spacings per distribution power grid type in FL1.05 of Fig. 3(a) of [1], no_1 and no_2, which are the number of spacings for the horizontal and vertical axis, respectively, should be assumed. In this paper, the number of spacings for the horizontal and vertical axis is assumed to be equal to 10 in both cases regardless of the distribution power grid type while this selection is going to be proven critical for the simulation time of class mapping (see Sec.3.5).

2. $m$ SHM definition procedure: In contrast with iSHM definition procedure, there is only one CASD that is used across the mSHM definition procedure, say Empirical CASD, but Empirical CASD is characterized by its CDF and not by corresponding MLEs [4]. In accordance with FL2.04 of Fig. 3(b) of [1], Empirical CDFs of the indicative distribution BPL topologies of main subclasses are computed. Conversely to iSHM, mSHM definition procedure demands the assumption of a reference indicative distribution BPL topology among the indicative ones so that suitable horizontal and vertical shifts can be added and the class map can be plotted. To compute the horizontal and vertical shift spacings in FL2.05 of Fig. 3(b) of [1] given the reference indicative distribution BPL topology, no_3 and no_4, which are the number of spacings for the horizontal and vertical axis, respectively, should be assumed. In this paper, the number of spacings for the horizontal and vertical axis is assumed to be equal to 10 in both cases regardless of the distribution power grid type examined. At this step of the definition procedure, same number of horizontal and vertical spacings is considered between iSHM and mSHM definition procedures for duration comparison reasons. Note that the maximum and minimum horizontal shift is assumed to be equal to $30 \mathrm{~dB}$ and $-30 \mathrm{~dB}$, respectively, while the maximum and minimum vertical shift is assumed to be equal to 1 and 0 , respectively.

Since all the required assumptions concerning the operation settings of iSHM, $\mathrm{mSHM}$ and definition procedure have been reported in this Section, the numerical results of iSHM and mSHM definition procedure are presented in the following Section for the OV MV and UN MV BPL topologies as well as the class maps. 


\section{Numerical Results and Discussion}

In this Section, numerical results concerning the definition procedure of iSHM and $\mathrm{mSHM}$ are presented. Taking into account the already identified default operation settings of Secs.2.1-2.4, four different scenarios concerning the class mapping are presented, namely: (i) iSHM definition procedure for OV MV BPL topologies; (ii) iSHM definition procedure for UN MV BPL topologies; (iii) $\mathrm{mSHM}$ definition procedure for OV MV BPL topologies; and (iv) $\mathrm{mSHM}$ definition procedure for UN MV BPL topologies. This Section ends with observations concerning the simulation time of the aforementioned four scenarios.

\section{1 iSHM Definition Procedure for OV MV BPL topologies}

With reference to Table 3 of [3], Weibull CASD achieves the best capacity estimations in OV MV BPL topologies with average absolute percentage change that is equal to $0.47 \%$ and remains the smallest one among the five examined CASDs. For the five indicative OV MV BPL topologies of the main subclasses of Table 1 of [1], the respective $\hat{a}_{\mathrm{MLE}}^{\text {Weibull }}$ and $\hat{\beta}_{\mathrm{MLE}}^{\text {Weibull }}$, which are the Weibull CASD MLEs, are reported in Table 1 of [3] while the respective capacities are given in Table 3 of [3]. On the basis of the Weibull CASD MLEs and capacities of the five indicative OV MV BPL topologies of the main subclasses of Table 1 of [1], the spacings for the horizontal and vertical axis, as dictated by FL1.05 of Fig. 3(a) of [1], are equal to $\frac{19.40-1 \times 10^{-11}}{10}=1.94$ and $\frac{1.34-0}{10}=0.134$, respectively, while the capacity borders between the adjacent distribution BPL topology classes $C B o_{l}^{\mathrm{G}, \mathrm{C}}, l=1,2,3,4$, as dictated by FL1.03 of Fig. 3(a) of [1], are equal to $245 \mathrm{Mbps}$, 289Mbps, 332Mbps and 379Mbps, respectively. Summarizing the aforementioned analysis, the class map of OV MV BPL topologies is plotted in Fig. 1 with respect to $\hat{a}_{\mathrm{MLE}}^{\text {Weibull }}$ and $\hat{\beta}_{\mathrm{MLE}}^{\text {Weibull }}$ when the operation settings of Sec.2 are assumed. In the same 2D contour plot, the Weibull CASD MLEs of the five indicative OV MV BPL topologies of the main subclasses of Table 1 of [1] with the corresponding capacities are also shown. 


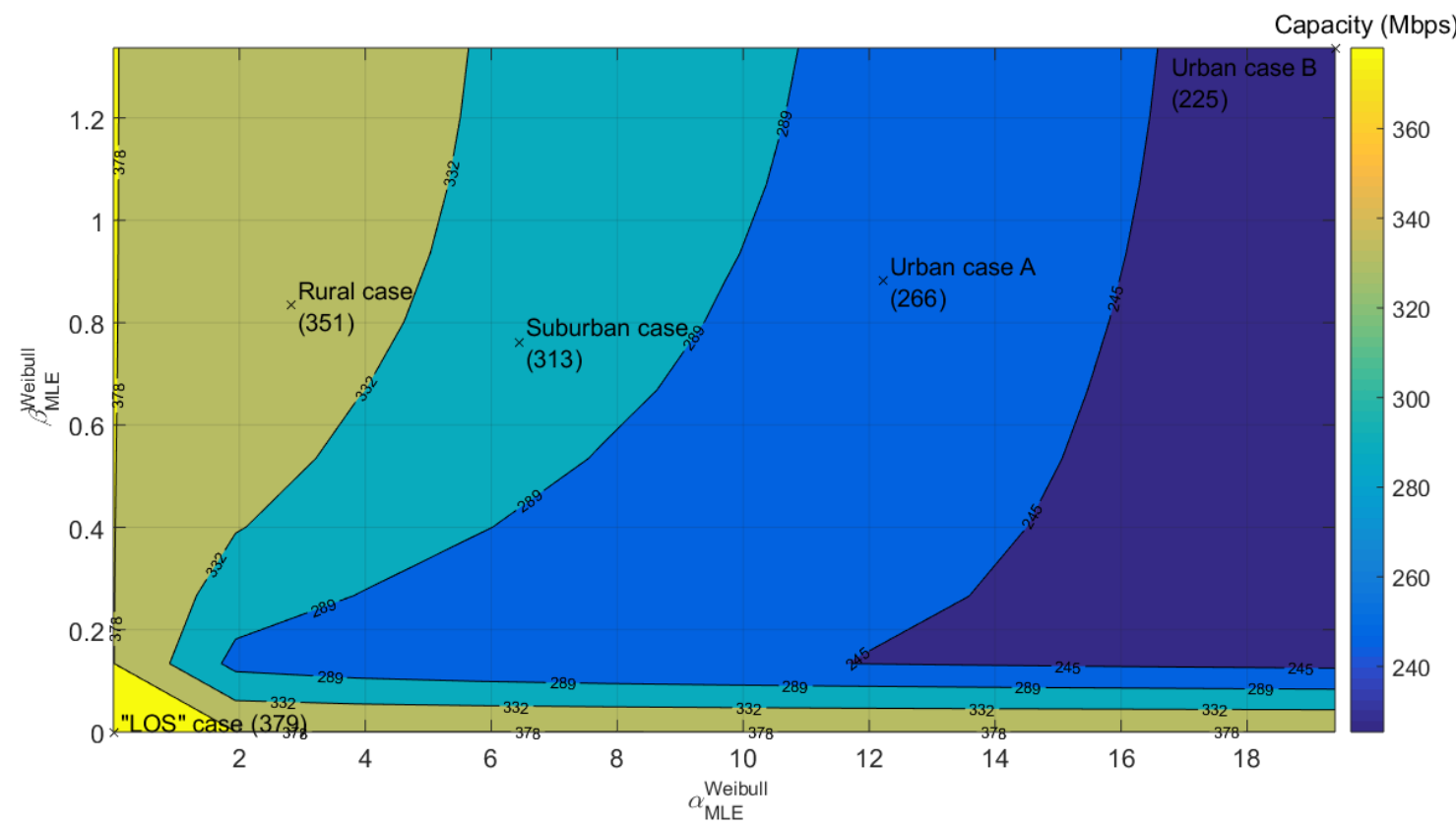

Fig. 1. iSHM class map of the OV MV BPL topologies in the $3-30 \mathrm{MHz}$ frequency band when $\mathrm{WtG}^{1}$ coupling scheme is deployed and FCC Part 15 is applied.

By observing Fig. 1, several useful findings can be pointed out for the class map of iSHM, namely:

- Different combinations of Weibull CASD MLEs entail different capacities for the corresponding virtual OV MV BPL topologies. With reference to the computed capacity borders between the adjacent distribution BPL topology classes, five capacity areas between these borders can be clearly delineated in the class map; say, the OV MV BPL "LOS" class, OV MV BPL rural class, OV MV BPL suburban class, OV MV BPL urban case A class and OV MV BPL urban case B class.

- As it is obvious, the indicative OV MV BPL topologies of the main subclasses of Table 1 of [1] are located in the homonymous title class areas. Note that the $\hat{\beta}_{\mathrm{MLE}}^{\text {Weibull }}$ value of the OV MV BPL "LOS" case has been arbitrarily chosen to be equal to zero since a narrow OV MV BPL "LOS" class area extents from the zero value up to the infinity of $\hat{\beta}_{\mathrm{MLE}}^{\text {Weibull }}$. Similarly, a narrow OV MV BPL "LOS" class area extents from the zero value up to the infinity of $\hat{a}_{\mathrm{MLE}}^{\text {Weibull }}$ creating a capacity fluctuation till the second $\hat{\beta}_{\mathrm{MLE}}^{\text {Weibull }}$ value of study (say, second $\hat{\beta}_{\mathrm{MLE}}^{\text {Weibull }}$ value is equal to 0.134). In mathematical terms, this is explained by the definition of the Weibull CASD CDF in eqs. (A10)-(A12) of [2] and the involvement of $\hat{a}_{\mathrm{MLE}}^{\text {Weibull }}$ and $\hat{\beta}_{\mathrm{MLE}}^{\mathrm{Weibull}}$.

- As the multipath environment of the examined OV MV BPL topologies becomes richer (i.e., more frequent and deeper spectral notches [5]-[6], [9], [41]), this has as a result the value increase of $\hat{a}_{\mathrm{MLE}}^{\text {Weibull }}$. Hence, OV MV BPL urban topologies tend to be located at the upper right areas of the class map while the OV MV BPL rural topologies tend to be located at the lower left areas. Anyway, $\hat{a}_{\mathrm{MLE}}^{\text {Weibull }}$ is 
more sensitive to the multipath environment aggravation in contrast with $\hat{\beta}_{\mathrm{MLE}}^{\text {Weibull }}$ that remains relatively insensitive. The last remark is validated by the fact that indicative OV MV BPL rural, OV MV BPL suburban and OV MV BPL urban case A topologies of Table 1 of [1] are characterized by approximately equal values of $\hat{\beta}_{\mathrm{MLE}}^{\text {Weibull }}$.

- Note that for given $\hat{a}_{\mathrm{MLE}}^{\text {Weibull }}$, the capacity of the virtual OV MV BPL topologies increases with respect to the $\hat{\beta}_{\mathrm{MLE}}^{\text {Weibull }}$. This is explained by studying Fig. (2) of [4], eq. (A10) of [2] and the behavior of Weibull CASD CDF; since $\hat{a}_{\mathrm{MLE}}^{\text {Weibull }}$ receives values that are significantly greater than 1 for the practical cases of interest, the term $\frac{\Delta A_{q}}{\hat{a}_{\mathrm{MLE}}^{W} \mathrm{Libull}}$ of eq. (10) of [2] can be considered to be greater than 1 in the majority of the cases examined in Fig. (2) of [4] where $\Delta A_{q}$ is the coupling scheme channel attenuation difference between the examined OV MV BPL topology and its respective "LOS" case at $q$ flat-fading subchannel. As $\hat{a}_{\mathrm{MLE}}^{\text {Weibull increases, }}-\left(\frac{\Delta A_{q}}{\hat{a}_{\mathrm{MLE}}^{\text {eibull }}}\right)^{\beta_{\mathrm{MLE}} \text { ieibull }}$ increases from -1 to infinity and, thus, $1-\exp \left[-\left(\frac{\Delta A_{q}}{\hat{a}_{M \text { eibull }}}\right)^{\beta_{\text {MLE }}^{\text {Weibull }}}\right]$ starts from $[1-\exp (-1)]$ and fast tends to 1 . The last observation has as a result the improvement of the capacities of the virtual OV MV BPL topologies that are characterized by greater $\hat{\beta}_{\mathrm{MLE}}^{\text {Weibull }}$ values for given $\hat{a}_{\mathrm{MLE}}^{\text {Weibull }}$. Anyway, the capacity improvement is more evident for the cases of low $\hat{a}_{\mathrm{MLE}}^{\mathrm{Weibull}}$ because the term $\frac{\Delta A_{q}}{\hat{a}_{\mathrm{MLE}}^{\mathrm{Webull}}}$ remains significantly greater than 1 and this explains the appearance of the class area edges at the bottom of the class map and the steep and almost vertical class area borders for $\hat{\beta}_{\mathrm{MLE}}^{\text {Weibull }}$ greater than 1.2 .

\section{2 iSHM Definition Procedure for UN MV BPL topologies}

With reference to Table 3 of [3], Wald CASD achieves the best capacity estimations in UN MV BPL topologies with average absolute percentage change that is equal to $0.01 \%$ and remains the smallest one among the five examined CASDs. For the five indicative UN MV BPL topologies of the main subclasses of Table 2 of [1], the respective $\hat{\mu}_{\mathrm{MLE}}^{\mathrm{Wald}}$ and $\hat{\lambda}_{\mathrm{MLE}}^{\mathrm{Wald}}$, which are the Wald CASD MLEs, are reported in Table 1 of [3] while the respective capacities are given in Table 3 of [3]. By observing the extent of $\hat{\lambda}_{\mathrm{MLE}}^{\text {Wald }}$ values of Table 2 of [1], which ranges from 20.93 to $2.62 \times 10^{3}$, and taking under consideration the number of spacings that is equal to 10 , the proper selection for the vertical axis is the representation of $\left(\hat{\lambda}_{\mathrm{MLE}}^{\text {Wald }}\right)^{-1}$ and the corresponding vertical spacings. Therefore, on the basis of the Wald CASD MLEs and capacities of the five indicative OV MV BPL topologies of the main subclasses of Table 2 of [1], the spacings for the horizontal and vertical axis, as dictated by FL1.05 of Fig. 3 (a) of [1], are equal to $\frac{19.96-1 \times 10^{-6}}{10}=1.996$ and $\frac{\left(\frac{1}{20.98}\right)-\left(\frac{1}{2.62 \times 10^{8}}\right)}{10}=0.0047$, respectively, while the capacity borders between the adjacent distribution BPL topology classes $C B o_{l}^{\mathrm{G}, \mathrm{C}}, l=1,2,3,4$, as dictated by FL1.03 of Fig. 3(a) of [1], are equal to $561 \mathrm{Mbps}$, 619Mbps, 651Mbps and 699Mbps, respectively. Summarizing the aforementioned analysis, the class map of UN MV BPL topologies is plotted in Fig. 2 with respect to 
$\hat{\mu}_{\mathrm{MLE}}^{\mathrm{Wald}}$ and $\left(\hat{\lambda}_{\mathrm{MLE}}^{\text {Wald }}\right)^{-1}$ when the operation settings of Sec.2 are assumed. In the same 2D contour plot, the Wald CASD MLEs of the five indicative UN MV BPL topologies of the main subclasses of Table 2 of [1] with the corresponding capacities are also shown.

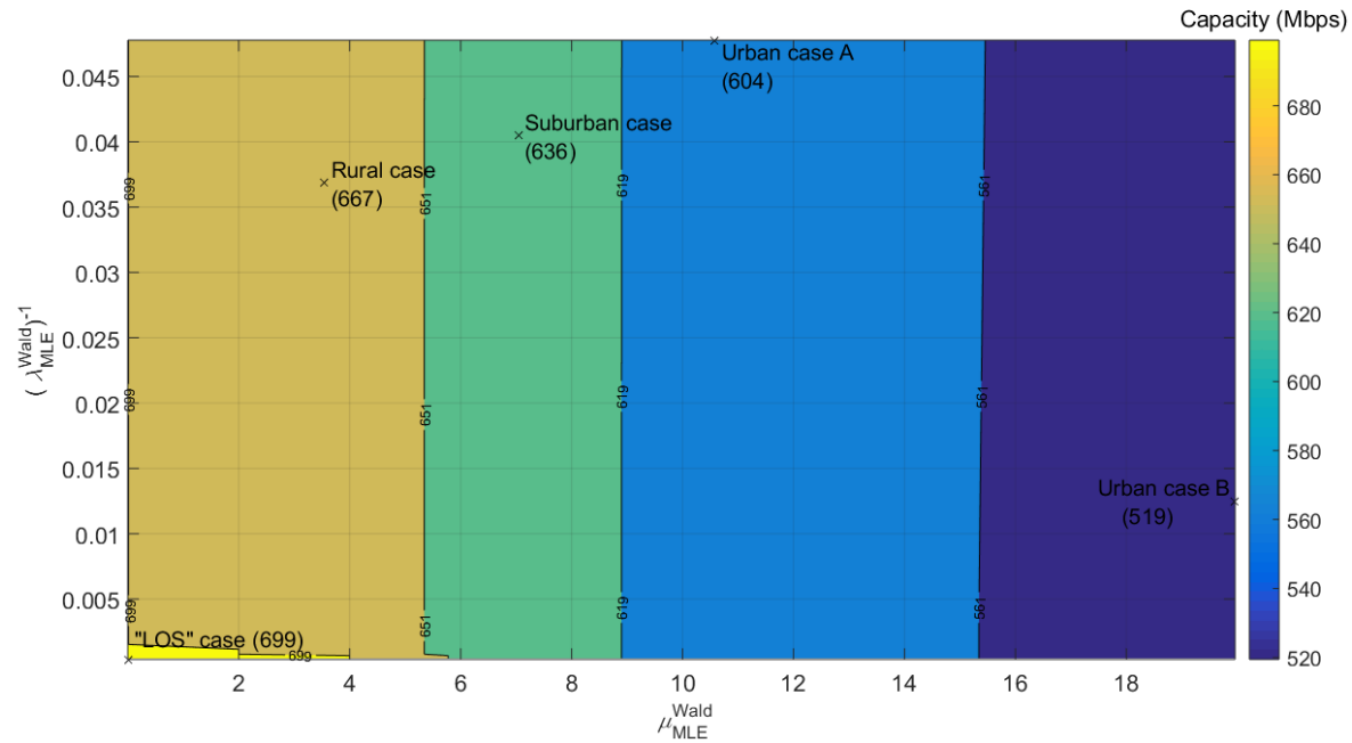

Fig. 2. iSHM class map of the UN MV BPL topologies in the $3-30 \mathrm{MHz}$ frequency band when $\mathrm{StP}^{1}$ coupling scheme is deployed and FCC Part 15 is applied.

By observing Fig. 2, additional findings for the class map of iSHM can be added to the ones of Sec. 3.1, namely:

- Similarly to the iSHM class map of OV MV BPL topologies, five capacity areas between the computed borders are clearly delineated in the iSHM class map of UN MV BPL topologies; say, the UN MV BPL "LOS” class, UN MV BPL rural class, UN MV BPL suburban class, UN MV BPL urban case A class and UN MV BPL urban case B class. Each of the five capacity areas consists of the homonymous indicative UN MV BPL topology of Table 2 of [1]. Also, in accordance with eq. (A7) of [2], a narrow UN MV BPL "LOS" class area extents from zero up to the infinity when $\hat{\mu}_{\mathrm{MLE}}^{\mathrm{Wald}}$ is equal to zero. This is explained by studying eq. (A7) of [2] and the behavior of Wald CASD CDF; since $\hat{\mu}_{\mathrm{MLE}}^{\text {Wald }}$ receives values that are significantly greater than 0 for the practical cases of interest, the terms where $\hat{\mu}_{\mathrm{MLE}}^{\mathrm{Wald}}$ is involved as a denominator receive values that are different than infinity. The zero theoretical value of $\hat{\mu}_{\mathrm{MLE}}^{\mathrm{Wald}}$ entails the special behaviors of the Wald CASD CDF and capacity regardless of the $\left(\hat{\lambda}_{\mathrm{MLE}}^{\mathrm{Wald}}\right)^{-1}$ value.

- As the multipath environment of the examined UN MV BPL topologies becomes richer (i.e., more frequent and deeper spectral notches [7], [9], [5], [6], [41]), this has as a result the value increase of $\hat{\mu}_{\mathrm{MLE}}^{\text {Wald }}$. Hence, UN MV BPL urban topologies tend to be located at the right areas of the class map while the UN MV BPL rural topologies tend to be located at the left areas. Similarly to the class map of OV 
MV BPL topologies, $\hat{\mu}_{\mathrm{MLE}}^{\text {Wald }}$ behaves similarly to $\hat{a}_{\mathrm{MLE}}^{\text {Weibull }}$ (i.e., CASD MLEs that are sensitive to the multipath environment aggravation) while $\left(\hat{\lambda}_{\mathrm{MLE}}^{\mathrm{Wald}}\right)^{-1}$ behaves similarly to $\hat{\beta}_{\mathrm{MLE}}^{\text {Weibull }}$ (i.e., CASD MLEs that do not depend on the multipath environment aggravation). Conversely to iSHM class map of OV MV BPL topologies, iSHM class map of UN MV BPL topologies does not comprise class area edges of the extent demonstrated in OV MV BPL topologies.

\section{3 mSHM Definition Procedure for OV MV BPL topologies}

With reference to Table 3 of [4], Empirical CASD of mSHM achieves better capacity estimations than the ones of Weibull CASD of iSHM in OV MV BPL topologies when OV MV BPL urban case A, OV MV BPL suburban case and OV MV BPL rural case are examined while the capacity estimation difference between Empirical and Weibull CASDs remains relatively small when OV MV BPL urban case B is examined. In total, Empirical CASD of mSHM achieves better average absolute percentage change (i.e., $0.09 \%$ ) than Weibull CASD of iSHM (i.e., 0.47\%).

In accordance with [1], similarly to the role of CASD selection of iSHM, the selection of the reference distribution BPL topology among the available indicative distribution BPL topologies of the main subclasses defines the class map of mSHM. In contrast with iSHM where one CASD excels over the others in terms of the capacity estimation performance (say, Weibull CASD and Wald CASD for the OV MV and UN MV BPL topologies, respectively) and is finally selected, all the four indicative OV MV BPL topologies of the main subclasses of Table 1 of [1], except for the OV MV "LOS" case, should be examined separately during the preparation of mSHM class maps.

For the reference indicative OV MV BPL urban case A of Table 1 of [1], the horizontal shift $h_{-}$shift $t_{1,1}^{\mathrm{G}, \mathrm{C}}$ and vertical shift $v_{-}$shift $t_{1,1}^{\mathrm{G}, \mathrm{C}}$ of its corresponding Empirical CDF, hereafter denoted simply as $h_{-}$shift and $v_{-}$shift, respectively, are assumed to be both equal to zero while the respective capacity is given in Table 3 of [3]. On the basis of the horizontal and vertical shifts of the Empirical CDF and the capacity of the reference indicative OV MV BPL urban case A, the spacing for the horizontal axis $\Delta h_{-} s h i f t_{1,1}^{\mathrm{G}, \mathrm{C}}$ and the spacing for the vertical axis $\Delta v_{-} \operatorname{shif} t_{1,1}^{\mathrm{G}, \mathrm{C}}$, as dictated by FL2.05 of Fig. 3(b) of [1], are equal to $\frac{30-(-30)}{10}=0.6$ and $\frac{1-0}{10}=0.1$, respectively, while the capacity borders between the adjacent distribution BPL topology classes $C B o_{l}^{\mathrm{G}, \mathrm{C}}, l=1,2,3,4$, as dictated by FL2.03 of Fig. 3(a) of [1], are equal to $245 \mathrm{Mbps}$, $289 \mathrm{Mbps}, 332 \mathrm{Mbps}$ and $379 \mathrm{Mbps}$, respectively. Note that the capacity borders between the adjacent distribution BPL topology classes remain the same during the design of iSHM and mSHM class maps. Summarizing the aforementioned analysis, the class map of OV MV BPL topologies is plotted in Fig. 3(a) with respect to the horizontal shift $h_{-}$shift and vertical shift $v_{-}$shift when the operation settings of Sec.2 are assumed. In the same 2D contour plot, the capacity borders between the adjacent distribution BPL topology classes and the capacity of the reference indicative OV MV BPL urban case A of Table 1 of [1] are also shown. In Figs. 3(b)-(d), same plots with Fig. 3(a) are given but for the case of the reference indicative urban case B, suburban case and rural case of Table 1 of [1], respectively. 


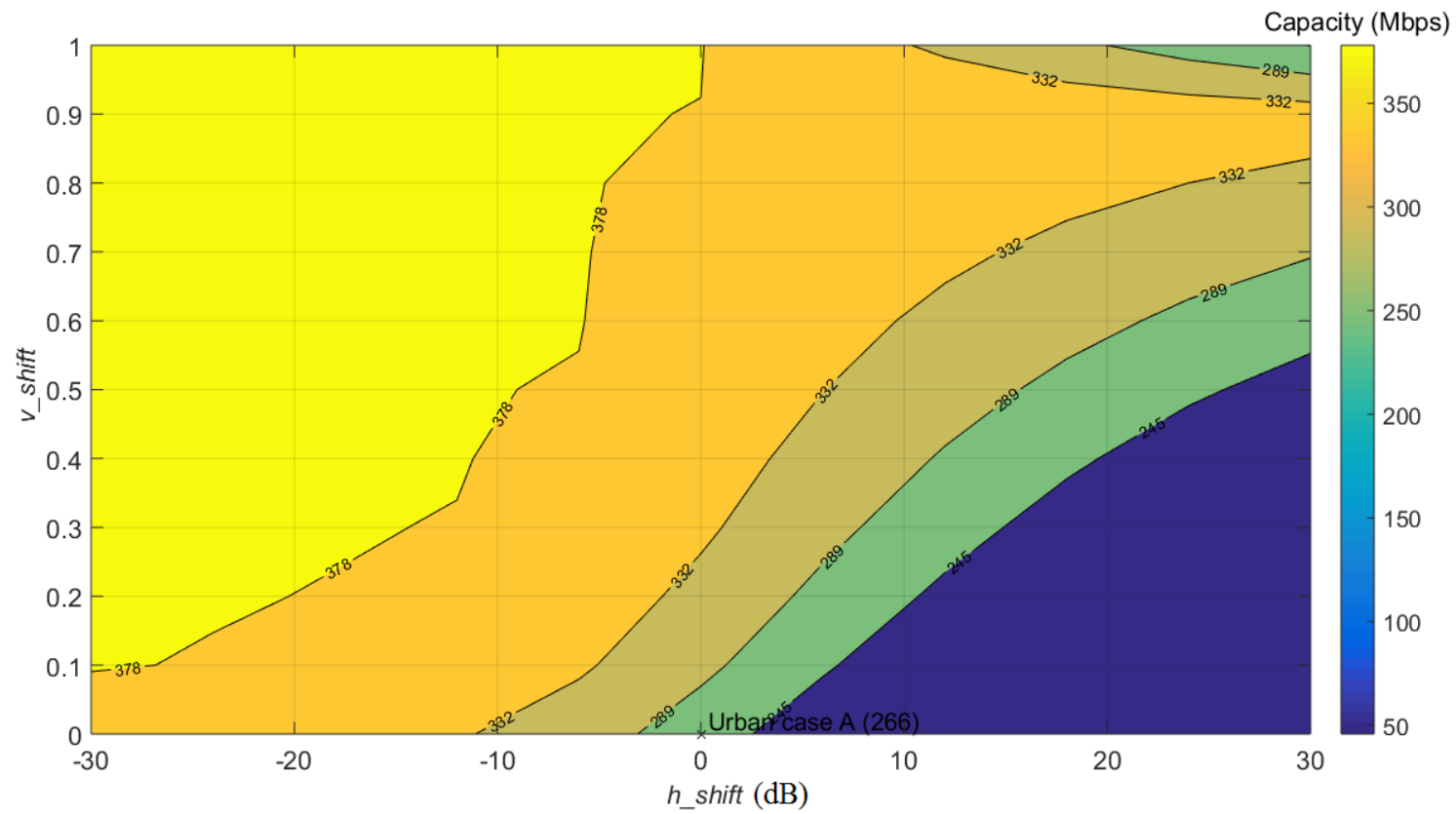

(a)

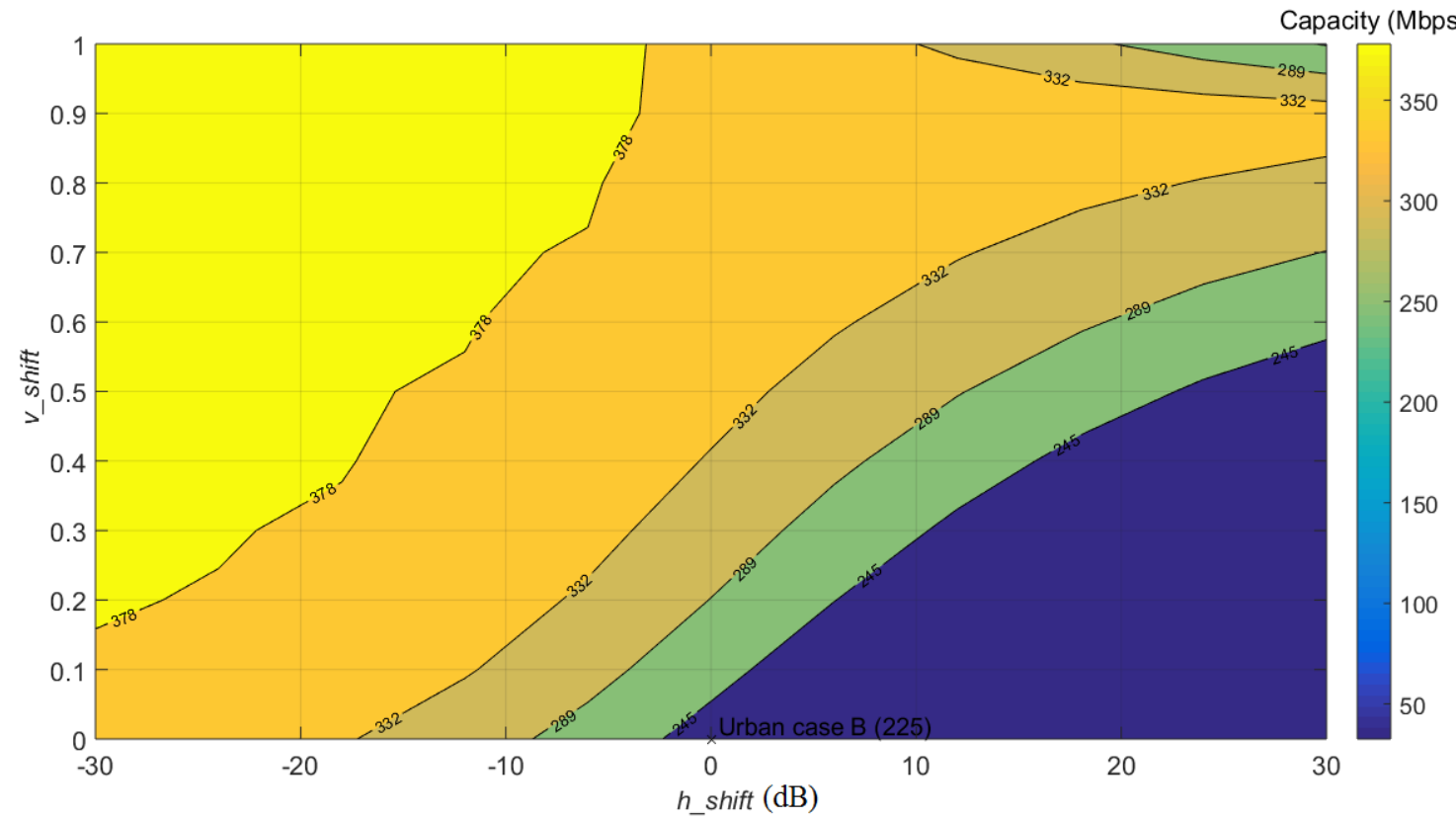

(b) 


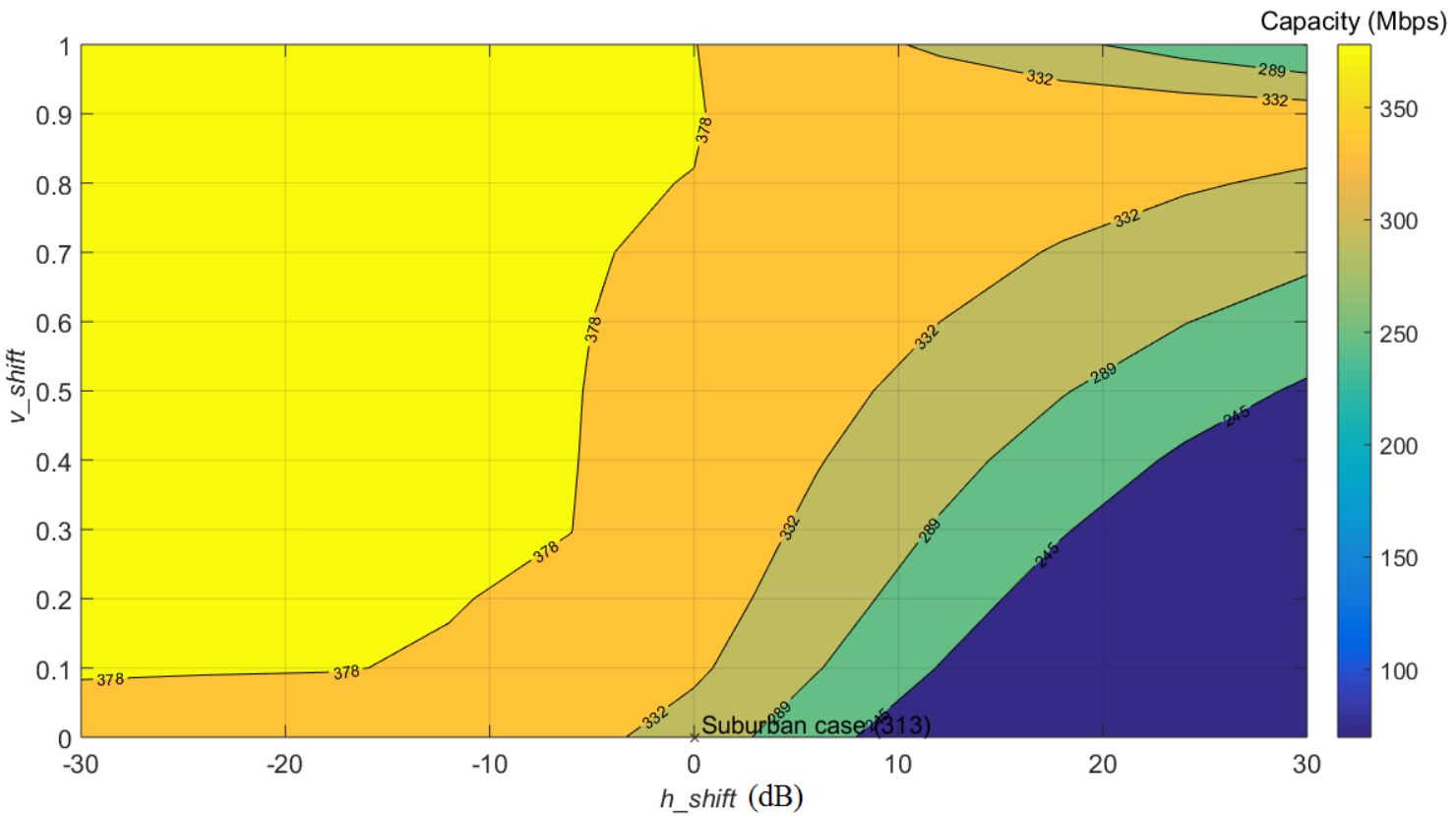

(c)

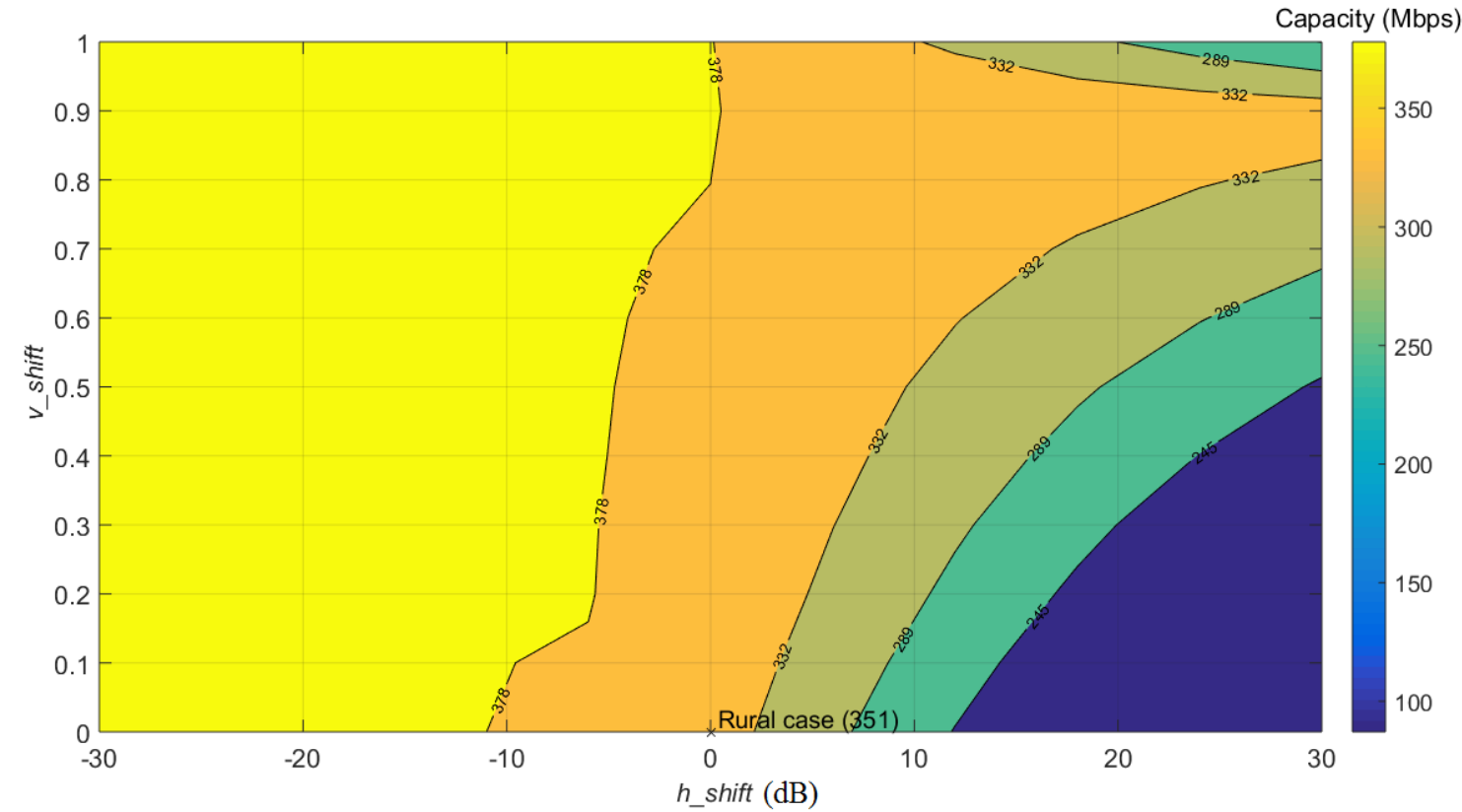

(d)

Fig. 3. mSHM class map of the OV MV BPL topologies in the $3-30 \mathrm{MHz}$ frequency band when $\mathrm{WtG}^{1}$ coupling scheme is deployed and FCC Part 15 is applied for different reference indicative OV MV BPL topologies. (a) OV MV BPL urban case A. (b) OV MV BPL urban case B. (c) OV MV BPL suburban case. (d) OV MV BPL rural case.

From Figs. 3(a)-(d), several interesting remarks can be pointed out concerning the mSHM class maps, namely:

- Similarly to the iSHM class map of OV MV BPL topologies, five capacity areas between the computed borders can be clearly delineated in the mSHM class map 
of OV MV BPL topologies; say, the OV MV BPL "LOS" class, OV MV BPL rural class, OV MV BPL suburban class, OV MV BPL urban case A class and UN MV BPL urban case B class. The virtual OV MV BPL topologies that are members of the aforementioned OV MV BPL topology classes can be defined by the suitable combined horizontal and vertical shift adjustment of the reference indicative OV MV BPL topology with reference to Figs. 3(a)-(d).

- As the procedure of the Empirical CDF shifting is concerned in this paper, the vertical shift is first taken into account and the horizontal shift is second executed. This sequence of shifts ensures that the shape of the Empirical CDF retains its characteristics as reported in eq.(11) of [1] and its accompanying two restrictions regarding the definition of valid shift pair combinations. Anyway, since the vertical and horizontal shifts can be considered as linear transformations of the Empirical CDFs, the sequence of shifts can be reversed without class map modifications if the new horizontal values are not modified during the vertical shifts.

- The vertical shifts that are assumed during the preparation of the mSHM class maps are considered to be positive and range from 0 to 1 while the horizontal shifts are assumed to range from $-30 \mathrm{~dB}$ to $30 \mathrm{~dB}$ regardless of the examined distribution BPL topology. As the vertical shifts are concerned, the positive values up to 1 , which are combined with the CDF maximum value restriction of 1 , imply that the virtual Empirical CDF that is produced after the valid shift pair combination reaches up to 1 . As the horizontal shifts are concerned, since coupling scheme channel attenuation differences of the reference indicative $\mathrm{OV}$ MV BPL topology take values greater than $1 \times 10^{-11} \mathrm{~dB}$, the negative horizontal shifts imply that the virtual OV MV BPL topology is characterized by lower channel attenuation than the one of the reference indicative OV MV BPL topology while the positive horizontal shifts imply the opposite result. Anyway, after the horizontal shift, the virtual coupling scheme channel attenuation difference always remains lower bounded by $1 \times 10^{-11} \mathrm{~dB}$.

- Regardless of the examined reference indicative OV MV BPL topology, higher capacities are observed in the upper left areas of the mSHM class map. Since restrictions concerning the virtual Empirical CDF and virtual coupling scheme channel attenuation differences have been already reported, the great areas of the OV MV BPL "LOS" topology class are observed in the upper left areas of the $\mathrm{mSHM}$ class map. As the examined reference indicative OV MV BPL topology is characterized by high capacity, there is no need for high boost of the Empirical CDF (i.e., vertical shift) so that the capacity of the virtual OV MV BPL topology reaches up to the capacity maximum that is the capacity of OV MV BPL "LOS" topology class. The latter explanation justifies the area expansion of the OV MV BPL "LOS" topology class up to the lower left areas of the mSHM class maps when OV MV BPL rural and OV MV BPL suburban topology classes are illustrated in Figs. 3(c) and 3(d), respectively.

- Regardless of the examined reference indicative OV MV BPL topology, lower capacities are observed especially in the lower right areas of the mSHM class map but also, in a certain extent, in upper right areas of the mSHM class map. As the lower right areas of the $\mathrm{mSHM}$ class map are examined, the high imposed 
coupling scheme channel attenuation differences by the high values of the horizontal shifts that are combined with the relatively low increase of Empirical CDF due to the low values of the vertical shifts normally reduce the capacities of the virtual OV MV BPL topologies. Here, there is no restriction to the imposed coupling scheme channel attenuation differences and, therefore, the capacities of the virtual OV MV BPL topologies tend to zero as the horizontal shifts significantly increase. As the upper right of the mSHM class map, which can be treated as a special case of the low capacity behavior, are investigated, the capacities of the virtual OV MV BPL topologies are observed to be decreased but remain higher than the capacities observed in the lower right areas of the mSHM class map when vertical shifts exceed 0.9. The behavior of the capacities of the virtual OV MV BPL topologies in the upper right areas of the mSHM class map is explained by the fact that, for given the maximum vertical shift (e.g., 1), as the horizontal shift increases above 0dB this implies that the virtual Empirical CDF is characterized by a step function of magnitude 1 while the step position is located at the examined horizontal step value. Due to the shape of the virtual Empirical $\mathrm{CDF}$, the virtual coupling scheme channel attenuation difference is fixed and equal to the examined horizontal step value. As the examined horizontal shift increases, so does the fixed virtual coupling scheme channel attenuation difference, thus having an effect of the reduction of the capacity of the examined virtual OV MV BPL topology.

- By identifying the five capacity areas between the computed borders in each of the mSHM class maps of Figs. 3(a)-(d), it is obvious that a plethora of virtual OV MV BPL topologies can enrich the different OV MV BPL topology classes by adopting different reference indicative OV MV BPL topologies when suitable combined horizontal and vertical shift adjustments that comply with the respective mSHM class map areas and Empirical CDFs are followed.

\section{4 mSHM Definition Procedure for UN MV BPL topologies}

With reference to Table 3 of [4], Empirical CASD of mSHM does not achieve better capacity estimations than the ones of Wald CASD of iSHM in UN MV BPL topologies but the percentage change differences in all the examined cases remain significantly low. Anyway, the average absolute percentage change between the Empirical CASD of mSHM (i.e., 0.07\%) and Wald CASD of iSHM (i.e., 0.01\%) again remains significantly low while the main advantage of the Empirical CASD against Wald CASD remains its execution time (as reported in Sec.3.5).

In accordance with [1] and similarly to Sec.3.3, all the four indicative UN MV BPL topologies of the main subclasses of Table 2 of [1], except for the UN MV "LOS" case, are examined separately during the preparation of mSHM class maps. As the horizontal shifts, vertical shifts, horizontal shift spacings and vertical shift spacings of the mSHM class maps of UN MV BPL topologies are considered, these are assumed to receive the same values with the respective ones of the mSHM class maps of OV MV BPL topologies. Also, the capacity borders between the adjacent distribution BPL topology classes $C B o_{l}^{\mathrm{G}, \mathrm{C}}, l=1,2,3,4$, which are adopted during the preparation of the mSHM class maps, are equal to 561 Mbps, 619 Mbps, 651 Mbps and 699 Mbps, 
respectively, and remain the same ones with the respective capacity borders during the preparation of the iSHM class maps already presented in Sec.3.2. The class map of UN MV BPL topologies is plotted in Fig. 4(a) with respect to the horizontal shift $h_{-}$shift and vertical shift $v_{-}$shift when the operation settings of Sec.2 are assumed. In the same 2D contour plot, the capacity borders between the adjacent distribution BPL topology classes and the capacity of the reference indicative UN MV BPL urban case A of Table 2 of [1] are also shown. In Figs. 4(b)-(d), same plots with Fig. 4(a) are given but for the case of the reference indicative UN MV BPL urban case B, UN MV BPL suburban case and UN MV BPL rural case of Table 2 of [1], respectively. 


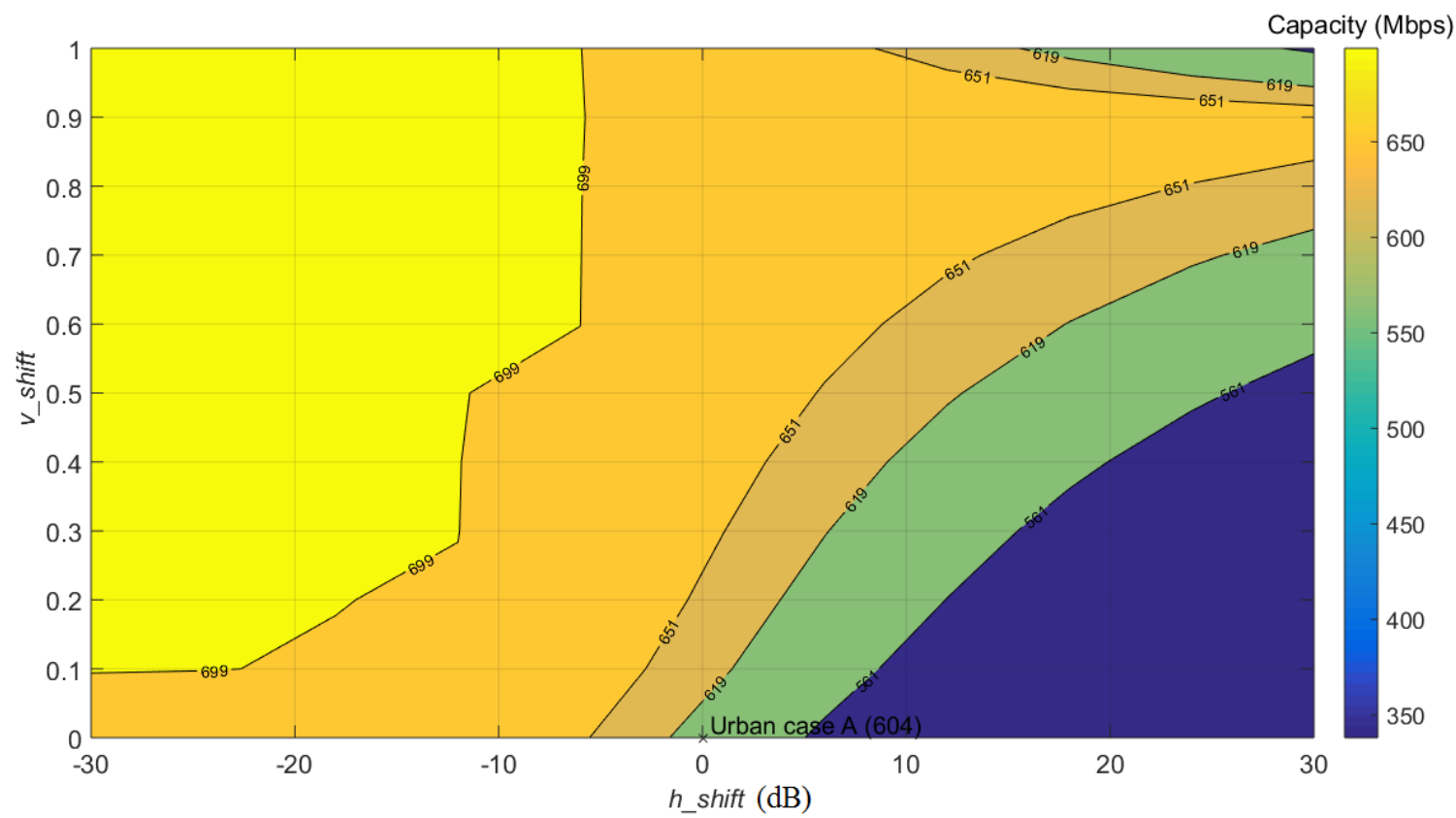

(a)

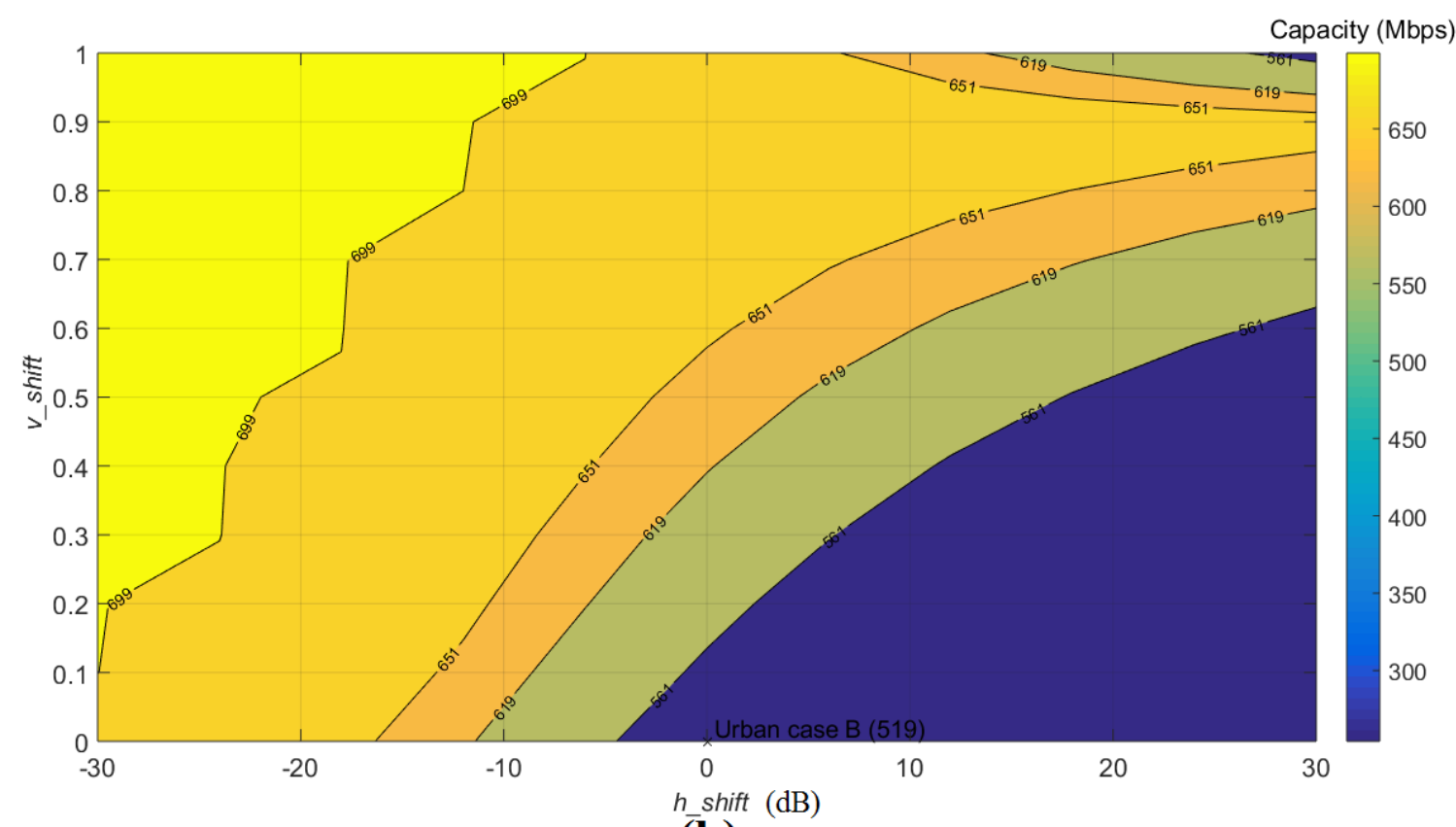

(b) 


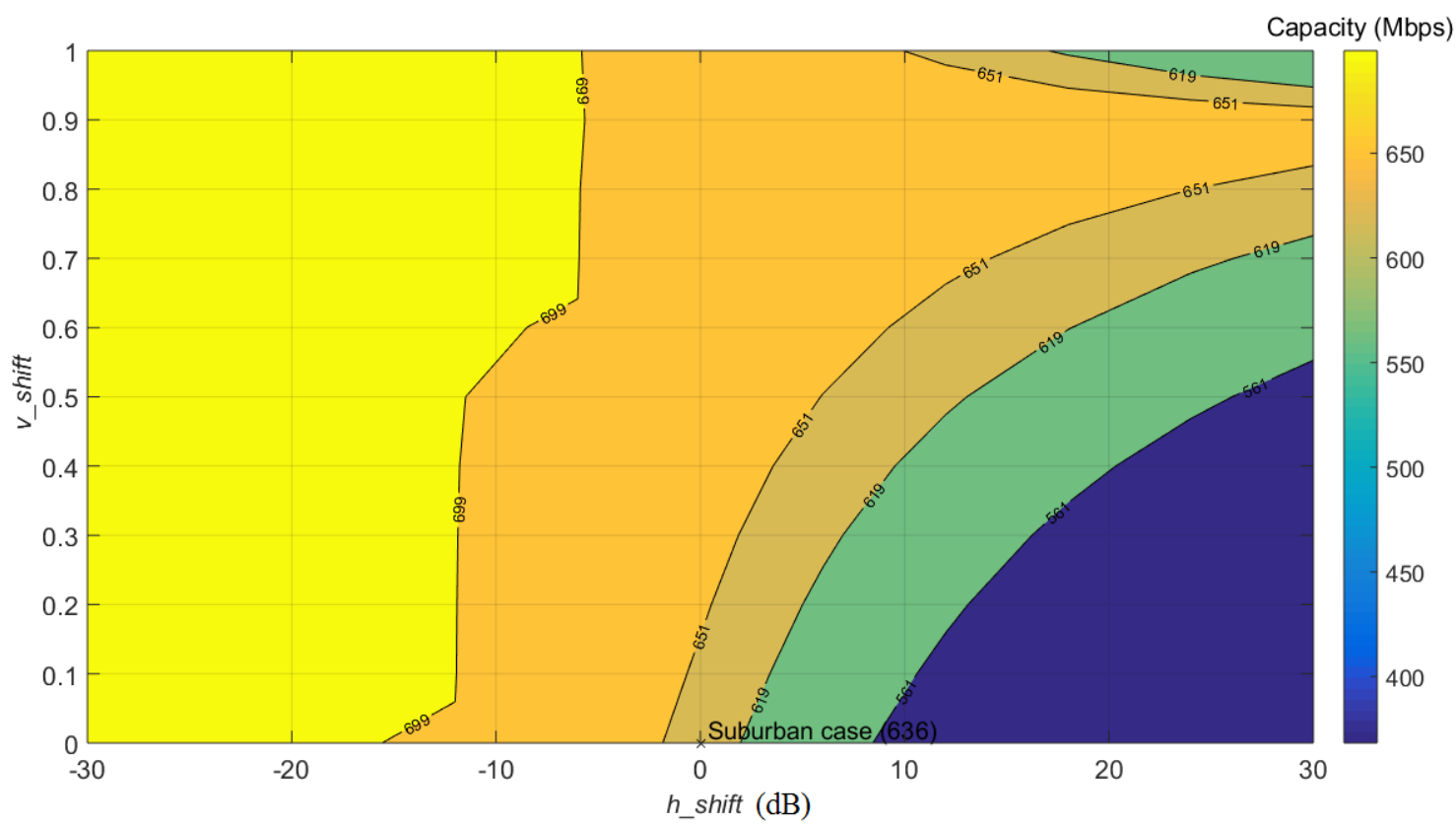

(c)

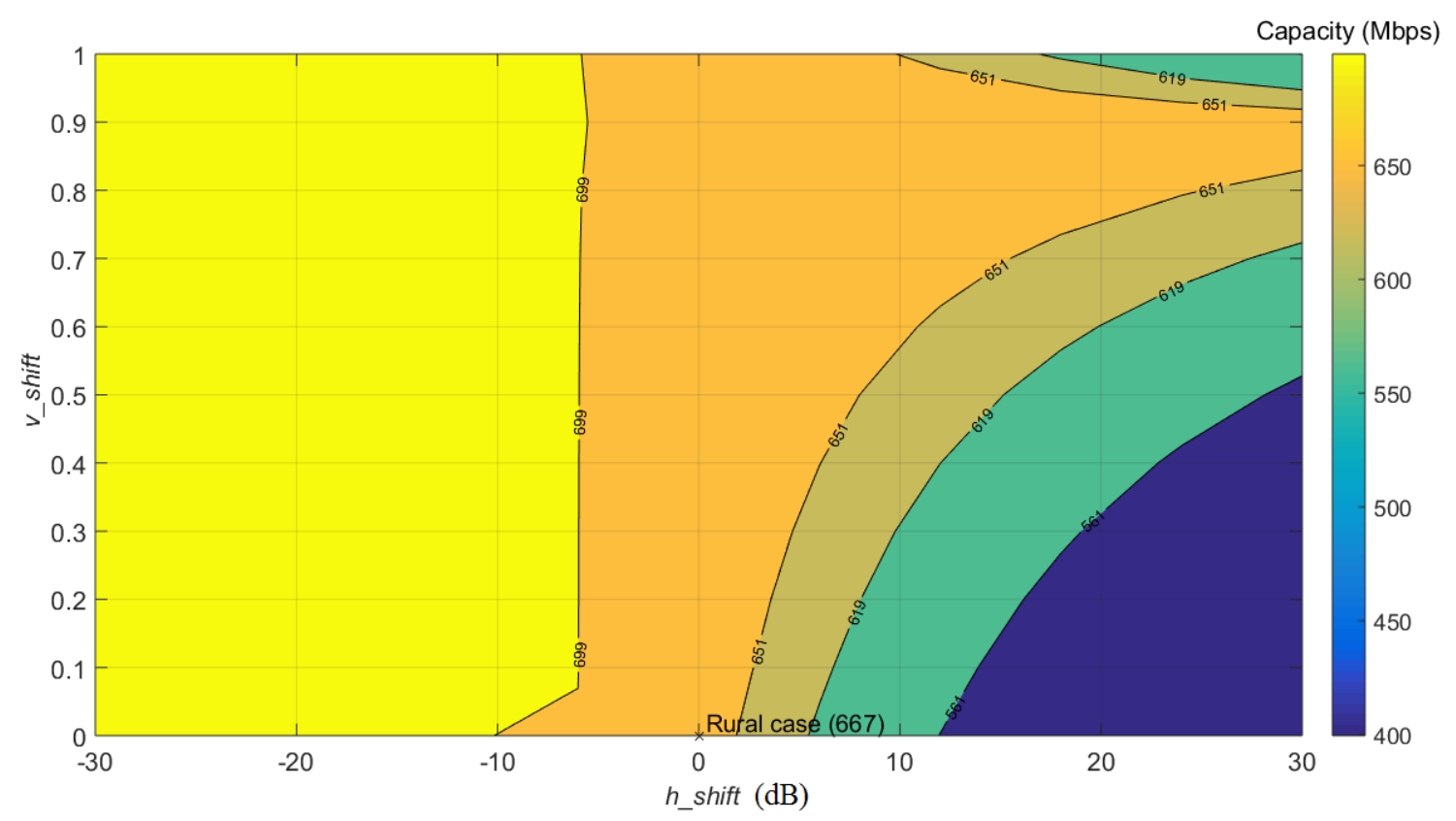

(d)

Fig. 4. mSHM class map of the UN MV BPL topologies in the $3-30 \mathrm{MHz}$ frequency band when $\mathrm{StP}^{1}$ coupling scheme is deployed and FCC Part 15 is applied for different reference indicative UN MV BPL topologies. (a) UN MV BPL urban case A. (b) UN MV BPL urban case B. (c) UN MV BPL suburban case. (d) UN MV BPL rural case.

By observing Figs. 4(a)-(d), several interesting conclusions can be reported concerning the mSHM class maps, namely:

- Similarly to the mSHM class maps of OV MV BPL topologies, five capacity areas between the computed borders are clearly delineated in the mSHM class 
map of UN MV BPL topologies whose planning remains the same as that of mSHM class maps of OV MV BPL topologies in Figs. 3(a)-(d).

- Similarly to mSHM class maps of the OV MV BPL topologies, the reference indicative UN MV BPL topology of each of the illustrated mSHM class maps is located at the axis center of each class map while the capacities of the virtual UN MV BPL topologies, which are defined with reference to the Empirical CDF of the reference indicative UN MV BPL topology, increase as the horizontal shift decreases (i.e., coupling scheme channel attenuation differences decrease) or the vertical shift increases (i.e., Empirical CDF shifts upward).

- By comparing the OV MV BPL "LOS" class areas of Figs. 3(a)-(d) against the UN MV BPL "LOS" class areas of Figs. 4(a)-(d), differences concerning the location of the right borderline of these areas, which are more clear when high values of vertical shifts are adopted, are here mentioned. By comparing Figs. 2 and 3 of [4], the coupling scheme channel attenuation differences of the UN MV BPL topologies are characterized by a fixed difference (i.e., the minimum of the coupling scheme channel attenuation difference remains above a channel attenuation difference threshold) across the examined frequency range in contrast with the coupling scheme channel attenuation differences of the OV MV BPL topologies whose minima are equal to zero. This behavior of the coupling scheme channel attenuation differences of the UN MV BPL topologies is reflected on the class maps where the upper right UN MV BPL "LOS" class area borderline is located at the horizontal shift that is equal to the aforementioned channel attenuation difference threshold. As it is evident this channel attenuation difference threshold is included across the entire right UN MV BPL "LOS" class area borderline which is not so evident when the vertical shift is significantly lower than 1.

- Similarly to the mSHM class map of OV MV BPL topologies, by identifying the five capacity areas between the computed borders in each of the mSHM class maps of Figs. 4(a)-(d), it is obvious that a plethora of virtual UN MV BPL topologies can again be defined in order to enrich the different UN MV BPL topology classes. By selecting among the different reference indicative OV MV BPL topologies and applying suitable combined horizontal and vertical shift adjustments in compliance with the respective mSHM class maps, virtual UN MV BPL topologies of different Empirical CDF forms that anyway are members of the same UN MV BPL topology class can be defined.

\subsection{Class Mapping and Simulation Time}

With reference to Table 3 of [4], different CASDs and power grid types are characterized by different capacity estimation performances (i.e., different percentage and average absolute percentage changes). Apart from the different capacity estimation performances, class mapping of Secs. 3.1-3.4 requires different simulation times that depend primarily on the applied CASD and secondarily on the power grid type given the operation settings of Sec. 2. More analytically, in Table 1, the required simulation times for the class mapping of Secs. 3.1-3.4, which have been recorded during the class map implementation, are reported. 
Table 1

The Simulation Time of iSHM and mSHM Class Maps

\begin{tabular}{|c|c|c|c|c|}
\hline SHM Type & $\begin{array}{c}\text { Power Grid } \\
\text { Type }\end{array}$ & CASD & $\begin{array}{c}\text { Simulation Time } \\
(\mathbf{s})\end{array}$ & $\begin{array}{c}\text { Paper Section and } \\
\text { Class Map Figure }\end{array}$ \\
\hline \multirow{2}{*}{ iSHM } & OV MV & Weibull & 407 & Sec. 3.1 / Fig. 1 \\
\cline { 2 - 5 } & UN MV & Wald & 22,137 & Sec. 3.2 / Fig. 2 \\
\hline \multirow{2}{*}{ mSHM } & OV MV & Empirical & 817 & Sec. 3.3 / Figs. 3(a) \\
\cline { 2 - 5 } & UN MV & Empirical & 758 & Sec. 3.4 / Figs. 4(a) \\
\hline
\end{tabular}

By combining the findings of Secs. 3.1-3.4 and the simulation time data of Table 1, several useful conclusions can be expressed regarding the trade-off between the performance and the speed of iSHM and mSHM CASDs, more specifically:

- As the Empirical CASD is the only CASD that is applied by mSHM, the simulation time that is required for the class mapping of OV MV and UN MV BPL topologies remains approximately the same (i.e., from $758 \mathrm{~s}$ to $817 \mathrm{~s}$ ). The small difference between the OV MV and UN MV BPL topology simulation times comes from the operation of the deterministic hybrid model. This is an evident outcome since the mSHM BPMN diagram of Fig. 2(b) of [1] and the $\mathrm{mSHM}$ definition procedure flowchart of Fig. 3(b) of [1] anyway remain the same regardless of the examined power grid type.

- With respect to the capacity estimation performance, different CASDs are applied by iSHM in order to create the class maps of OV MV and UN MV BPL topologies. Actually, Weibull and Wald CASDs are the suitable CASDs for the OV MV and UN MV BPL topologies, respectively, while the complexity of the respective MLE computation, as indicated in Secs. A.4 and A.3 of [2], differs. The different complexity of Weibull and Wald CASD MLE computation is reflected on the different simulation times of Table 1 for the iSHM class maps of OV MV and UN MV BPL topologies. In fact, with reference to Sec. A.3 of [2], the computation of Wald CASD CDF by twice using the Gaussian CDF $\Phi\{$. (see eq. A7 of [2]) skyrockets the required simulation time for the class mapping of UN MV BPL topologies (i.e., approximately 6.15 hours for iSHM class map of UN MV BPL topologies against approximately 7 min for iSHM class map of OV MV BPL topologies).

- Apart from the applied CASD and the power grid type, the simulation times, which are presented in Table 1, critically depend on the number of spacings, which remains the critical parameter of the operation settings presented in Sec. 2. At this moment, the number of spacings for the horizontal and vertical axes for iSHM and mSHM is assumed to be equal to 10 . This implies that $(10+1) \times(10+1)=121$ different MLE pair combinations and shift pair combinations are evaluated for the preparation of iSHM and mSHM class maps, respectively, while the required simulation times are presented in Table 1. If the number of spacings for the horizontal and vertical axes increases to 100, $(100+1) \times(100+1)=10,201$ different MLE pair combinations and shift pair combinations are required for the preparation of iSHM and mSHM class maps, 
respectively,

while the simulation times are expected to be increased by approximately 84 times. By analyzing the simulation time data of Table 1, this becomes a prohibitive task for the iSHM class mapping of UN MV BPL topologies (i.e., the simulation time is expected to be equal to approximately 21.5 days).

- By considering the capacity estimation performance and the simulation data of the different CASDs for the class mapping of UN MV BPL topologies, an interesting trade-off can be established between Empirical CASD of mSHM and the Wald CASD of iSHM. Although the capacity estimation performance of Wald CASD of iSHM presents slightly improved results with respect to the capacity estimation performance in relation with the Empirical CASD of mSHM, the simulation time of Wald CASD is six times greater than the one of Empirical CASD. Therefore, the slight improved capacity estimation performance is exchanged at significantly worse simulation times. Conversely, the same trade-off issue holds in OV MV BPL topologies between the Empirical CASD of mSHM and the Weibull CASD of iSHM.

By concluding this Section, it is evident that the definition of class maps of distribution BPL topologies can successfully enrich the existing distribution BPL topology classes with a plethora of statistically equivalent virtual distribution BPL topologies. However, the selection among different CASDs and different SHM types offers a diversity regarding the capacity estimation performance and the simulation time.

\section{Conclusions}

In this paper, the numerical results concerning the class mapping of OV MV and UN MV BPL topologies have been presented on the basis of iSHM and mSHM flowcharts and definition procedures, which have been analyzed in [1]. In accordance with the proposed class maps, it has been verified that distribution BPL topology classes can be further enriched with respective distribution BPL topology subclasses that further consist of a plethora of corresponding distribution BPL topologies that remain statistically equivalent with the indicative distribution BPL topology of the examined subclass. Apart from the definition of virtual distribution BPL topologies in terms of their capacity by simply selecting appropriate CASD parameters, the capacity estimation performance and the simulation time of iSHM and mSHM CASDs have been examined revealing an interesting trade-off between the aforementioned two parameters. After the class map definition, the statistical approach of SHM can be considered to be more robust since a great number of indicative distribution BPL topologies, which can act as representative topologies of respective distribution BPL topology subclasses, can be assumed.

\section{CONFLICTS OF INTEREST}

The author declares that there is no conflict of interests regarding the publication of this paper. 


\section{References}

[1] A. G. Lazaropoulos, "Virtual Indicative Broadband over Power Lines Topologies for Respective Subclasses by Adjusting Channel Attenuation Statistical Distribution Parameters of Statistical Hybrid Models - Part 1: Theory," Trends in Renewable Energy, vol. 5, no. 3, pp 237-257, Aug. 2019. DOI: 10.17737/tre.2019.5.3.0099

[2] A. G. Lazaropoulos, "Statistical Broadband over Power Lines Channel Modeling - Part 1: The Theory of the Statistical Hybrid Model," Progress in Electromagnetics Research C, vol. 92, pp. 1-16, 2019. [Online]. Available: http://www.jpier.org/PIERC/pierc92/01.19012902.pdf

[3] A. G. Lazaropoulos, "Statistical Broadband over Power Lines (BPL) Channel Modeling - Part 2: The Numerical Results of the Statistical Hybrid Model," Progress in Electromagnetics Research C, vol. 92, pp. 17-30, 2019. [Online]. Available: http://www.jpier.org/PIERC/pierc92/02.19012903.pdf

[4] A. G. Lazaropoulos, "Enhancing the Statistical Hybrid Model Performance in Overhead and Underground Medium Voltage Broadband over Power Lines Channels by Adopting Empirical Channel Attenuation Statistical Distribution," Trends in Renewable Energy, vol. 5, no. 2, pp. 181-217, 2019. [Online]. Available: http://futureenergysp.com/index.php/tre/article/view/96/pdf

[5] A. G. Lazaropoulos, "Towards Modal Integration of Overhead and Underground Low-Voltage and Medium-Voltage Power Line Communication Channels in the Smart Grid Landscape: Model Expansion, Broadband Signal Transmission Characteristics, and Statistical Performance Metrics (Invited Paper)," ISRN Signal Processing, vol. 2012, Article ID 121628, pp. 1-17, 2012. [Online]. Available: http://www.hindawi.com/isrn/sp/2012/121628/

[6] A. G. Lazaropoulos, "Towards Broadband over Power Lines Systems Integration: Transmission Characteristics of Underground Low-Voltage Distribution Power Lines," Progress in Electromagnetics Research B, vol. 39, pp. 89-114, 2012. [Online]. Available: http://www.jpier.org/PIERB/pierb39/05.12012409.pdf

[7] A. G. Lazaropoulos and P. G. Cottis, "Transmission characteristics of overhead medium voltage power line communication channels," IEEE Trans. Power Del., vol. 24, no. 3, pp. 1164-1173, Jul. 2009.

[8] A. G. Lazaropoulos and P. G. Cottis, "Capacity of overhead medium voltage power line communication channels," IEEE Trans. Power Del., vol. 25, no. 2, pp. 723-733, Apr. 2010.

[9] A. G. Lazaropoulos and P. G. Cottis, "Broadband transmission via underground medium-voltage power lines-Part I: transmission characteristics," IEEE Trans. Power Del., vol. 25, no. 4, pp. 2414-2424, Oct. 2010.

[10] A. G. Lazaropoulos and P. G. Cottis, "Broadband transmission via underground medium-voltage power lines-Part II: capacity," IEEE Trans. Power Del., vol. 25, no. 4, pp. 2425-2434, Oct. 2010.

[11] A. G. Lazaropoulos, "Broadband transmission and statistical performance properties of overhead high-voltage transmission networks," Hindawi Journal of 
Computer Networks and Commun., 2012, article ID 875632, 2012. [Online]. Available: http://www.hindawi.com/journals/jcnc/aip/875632/

[12] P. Amirshahi and M. Kavehrad, "High-frequency characteristics of overhead multiconductor power lines for broadband communications," IEEE J. Sel. Areas Commun., vol. 24, no. 7, pp. 1292-1303, Jul. 2006.

[13] T. Sartenaer, "Multiuser communications over frequency selective wired channels and applications to the powerline access network" Ph.D. dissertation, Univ. Catholique Louvain, Louvain-la-Neuve, Belgium, Sep. 2004.

[14] T. Calliacoudas and F. Issa, "'Multiconductor transmission lines and cables solver," An efficient simulation tool for plc channel networks development," presented at the IEEE Int. Conf. Power Line Communications and Its Applications, Athens, Greece, Mar. 2002.

[15] T. Sartenaer and P. Delogne, "Deterministic modelling of the (Shielded) outdoor powerline channel based on the multiconductor transmission line equations," IEEE J. Sel. Areas Commun., vol. 24, no. 7, pp. 1277-1291, Jul. 2006.

[16] A. G. Lazaropoulos, "Virtual Indicative Broadband over Power Lines Topologies for Respective Subclasses by Adjusting Channel Attenuation Statistical Distribution Parameters of Statistical Hybrid Models - Part 3: The Case of Overhead Transmission Power Grids," Trends in Renewable Energy, vol. 5, no. 3, pp 282-306, Aug. 2019. DOI: 10.17737/tre.2019.5.3.00101

[17] S. Liu and L. J. Greenstein, "Emission characteristics and interference constraint of overhead medium-voltage broadband power line (BPL) systems," in Proc. IEEE Global Telecommunications Conf., New Orleans, LA, USA, Nov./Dec. 2008, pp. 1-5.

[18] A. G. Lazaropoulos, "Underground Distribution BPL Connections with $(\mathrm{N}+1)$ hop Repeater Systems: A Novel Capacity Mitigation Technique," Elsevier Computers and Electrical Engineering, vol. 40, pp. 1813-1826, 2014.

[19] A. G. Lazaropoulos, "Review and Progress towards the Capacity Boost of Overhead and Underground Medium-Voltage and Low-Voltage Broadband over Power Lines Networks: Cooperative Communications through Two- and ThreeHop Repeater Systems," ISRN Electronics, vol. 2013, Article ID 472190, pp. 119, 2013. [Online]. Available: http://www.hindawi.com/isrn/electronics/aip/472190/

[20] A. G. Lazaropoulos, "Broadband over Power Lines (BPL) Systems Convergence: Multiple-Input Multiple-Output (MIMO) Communications Analysis of Overhead and Underground Low-Voltage and Medium-Voltage BPL Networks (Invited Paper)," ISRN Power Engineering, vol. 2013, Article ID 517940, pp. 1-30, 2013. [Online].

Available: http://www.hindawi.com/isrn/power.engineering/2013/517940/

[21] A. G. Lazaropoulos, "Deployment Concepts for Overhead High Voltage Broadband over Power Lines Connections with Two-Hop Repeater System: Capacity Countermeasures against Aggravated Topologies and High Noise Environments," Progress in Electromagnetics Research B, vol. 44, pp. 283-307, 2012. [Online]. Available: http://www.jpier.org/PIERB/pierb44/13.12081104.pdf

[22] N. Suljanović, A. Mujčić, M. Zajc, and J. F. Tasič, "Approximate computation of high-frequency characteristics for power line with horizontal disposition and 
middle-phase to ground coupling," Elsevier Electr. Power Syst. Res., vol. 69, pp. 17-24, Jan. 2004.

[23] OPERA1, D5: Pathloss as a function of frequency, distance and network topology for various LV and MV European powerline networks. IST Integrated Project No 507667, Apr. 2005.

[24] N. Suljanović, A. Mujčić, M. Zajc, and J. F. Tasič, "High-frequency characteristics of high-voltage power line," in Proc. IEEE Int. Conf. on Computer as a Tool, Ljubljana, Slovenia, Sep. 2003, pp. 310-314.

[25] N. Suljanović, A. Mujčić, M. Zajc, and J. F. Tasič, "Power-line high-frequency characteristics: analytical formulation," in Proc. Joint 1st Workshop on Mobile Future \& Symposium on Trends in Communications, Bratislava, Slovakia, Oct. 2003, pp. 106-109.

[26] W. Villiers, J. H. Cloete, and R. Herman, "The feasibility of ampacity control on HV transmission lines using the PLC system," in Proc. IEEE Conf. Africon, George, South Africa, Oct. 2002, vol. 2, pp. 865-870.

[27] P. Amirshahi, "Broadband access and home networking through powerline networks" Ph.D. dissertation, Pennsylvania State Univ., University Park, PA, May 2006.

[28] OPERA1, D44: Report presenting the architecture of plc system, the electricity network topologies, the operating modes and the equipment over which PLC access system will be installed, IST Integr. Project No 507667, Dec. 2005.

[29] J. Anatory, N. Theethayi, R. Thottappillil, M. M. Kissaka, and N. H. Mvungi, "The influence of load impedance, line length, and branches on underground cable Power-Line Communications (PLC) systems," IEEE Trans. Power Del., vol. 23, no. 1, pp. 180-187, Jan. 2008.

[30] J. Anatory, N. Theethayi, and R. Thottappillil, "Power-line communication channel model for interconnected networks-Part II: Multiconductor system," IEEE Trans. Power Del., vol. 24, no. 1, pp. 124-128, Jan. 2009.

[31] J. Anatory, N. Theethayi, R. Thottappillil, M. M. Kissaka, and N. H. Mvungi, "The effects of load impedance, line length, and branches in typical low-voltage channels of the BPLC systems of developing countries: transmission-line analyses," IEEE Trans. Power Del., vol. 24, no. 2, pp. 621-629, Apr. 2009.

[32] T. Banwell and S. Galli, "A novel approach to accurate modeling of the indoor power line channel-Part I: Circuit analysis and companion model," IEEE Trans. Power Del., vol. 20, no. 2, pp. 655-663, Apr. 2005.

[33] W. Villiers, J. H. Cloete, L. M. Wedepohl, and A. Burger, "Real-time sag monitoring system for high-voltage overhead transmission lines based on powerline carrier signal behavior," IEEE Trans. Power Del., vol. 23, no. 1, pp. 389-395, Jan. 2008.

[34] A. G. Lazaropoulos, "Smart Energy and Spectral Efficiency (SE) of Distribution Broadband over Power Lines (BPL) Networks - Part 1: The Impact of Measurement Differences on SE Metrics," Trends in Renewable Energy, vol. 4, no. 2, pp. 125-184, Aug. 2018. [Online]. Available: http://futureenergysp.com/index.php/tre/article/view/76/pdf

[35] A. G. Lazaropoulos, "Broadband Performance Metrics and Regression Approximations of the New Coupling Schemes for Distribution Broadband over 
Power Lines (BPL) Networks," Trends in Renewable Energy, vol. 4, no. 1, pp. 43-73, Jan. $2018 . \quad$ [Online]. Available: http://futureenergysp.com/index.php/tre/article/view/59/pdf

[36] A. G. Lazaropoulos, "New Coupling Schemes for Distribution Broadband over Power Lines (BPL) Networks," Progress in Electromagnetics Research B, vol. 71, pp. 39-54, 2016. [Online]. Available: http://www.jpier.org/PIERB/pierb71/02.16081503.pdf

[37] A. G. Lazaropoulos, "A Panacea to Inherent BPL Technology Deficiencies by Deploying Broadband over Power Lines (BPL) Connections with Multi-Hop Repeater Systems," Bentham Recent Advances in Electrical \& Electronic Engineering, vol. 10, no. 1, pp. 30-46, 2017.

[38] A. G. Lazaropoulos, "The Impact of Noise Models on Capacity Performance of Distribution Broadband over Power Lines Networks," Hindawi Computer Networks and Communications, vol. 2016, Article ID 5680850, 14 pages, 2016. doi:10.1155/2016/5680850. [Online]. Available: http://www.hindawi.com/journals/jcnc/2016/5680850/

[39] A. G. Lazaropoulos, "Capacity Performance of Overhead Transmission MultipleInput Multiple-Output Broadband over Power Lines Networks: The Insidious Effect of Noise and the Role of Noise Models (Invited Paper)," Trends in Renewable Energy, vol. 2, no. 2, pp. 61-82, Jun. 2016. [Online]. Available: http://futureenergysp.com/index.php/tre/article/view/23

[40] A. G. Lazaropoulos, "Smart Energy and Spectral Efficiency (SE) of Distribution Broadband over Power Lines (BPL) Networks - Part 2: L1PMA, L2WPMA and L2CXCV for SE against Measurement Differences in Overhead Medium-Voltage BPL Networks," Trends in Renewable Energy, vol. 4, no. 2, pp. 185-212, Aug. 2018. [Online].

Available: http://futureenergysp.com/index.php/tre/article/view/77/pdf

[41] A. G. Lazaropoulos, "Factors Influencing Broadband Transmission Characteristics of Underground Low-Voltage Distribution Networks," IET Commun., vol. 6, no. 17, pp. 2886-2893, Nov. 2012.

Article copyright: (C) 2019 Athanasios G. Lazaropoulos. This is an open access article distributed under the terms of the Creative Commons Attribution 4.0 International License, which permits unrestricted use and distribution provided the original author and source are credited. 